\title{
Exploring the metabolomic diversity of plant species across spatial (leaf and stem) components and phylogenic groups
}

\author{
Sunmin Lee ${ }^{1}$, Dong-Gu Oh', Digar Singh', Jong Seok Lee ${ }^{2}$, Sarah Lee ${ }^{2^{*}}$ and Choong Hwan Lee ${ }^{1,3^{*}}$
}

\begin{abstract}
Background: Plants have been used as an important source of indispensable bioactive compounds in various cosmetics, foods, and medicines. However, the subsequent functional annotation of these compounds seems arduous because of the largely uncharacterized, vast metabolic repertoire of plant species with known biological phenotypes. Hence, a rapid multi-parallel screening and characterization approach is needed for plant functional metabolites.
\end{abstract}

Results: Fifty-one species representing three plant families, namely Asteraceae, Fabaceae, and Rosaceae, were subjected to metabolite profiling using gas chromatography time-of-flight mass spectrometry (GC-TOF-MS) and ultrahigh-performance liquid chromatography quadrupole orbitrap ion trap tandem mass spectrometry (UHPLC-Qorbitrap-MS/MS) as well as multivariate analyses. Partial least squares discriminant analysis (PLS-DA) of the metabolite profiling datasets indicated a distinct clustered pattern for 51 species depending on plant parts (leaves and stems) and relative phylogeny. Examination of their relative metabolite contents showed that the extracts from Fabaceae plants were abundant in amino acids, fatty acids, and genistein compounds. However, the extracts from Rosaceae had higher levels of catechin and ellagic acid derivatives, whereas those from Asteraceae were higher in kaempferol derivatives and organic acids. Regardless of the different families, aromatic amino acids, branch chain amino acids, chlorogenic acid, flavonoids, and phenylpropanoids related to the shikimate pathway were abundant in leaves. Alternatively, certain amino acids (proline, lysine, and arginine) as well as fatty acids levels were higher in stem extracts. Further, we investigated the associated phenotypes, i.e., antioxidant activities, affected by the observed spatial (leaves and stem) and intra-family metabolomic disparity in the plant extracts. Pearson's correlation analysis indicated that ellagic acid, mannitol, catechin, epicatechin, and quercetin derivatives were positively correlated with antioxidant phenotypes, whereas eriodictyol was positively correlated with tyrosinase inhibition activity.

Conclusions: This work suggests that metabolite profiling, including multi-parallel approaches and integrated bioassays, may help the expeditious characterization of plant-derived metabolites while simultaneously unraveling their chemodiversity.

Keywords: Plant parts, Chemodiversity, Antioxidant activity, Tyrosinase inhibition activity, Metabolite profiling

\footnotetext{
*Correspondence: Isr57@korea.kr; chlee123@konkuk.ac.kr

${ }^{2}$ National Institute of Biological Resources, Environmental Research Complex,

Incheon 22755, Korea

'Department of Bioscience and Biotechnology, Konkuk University, Seoul

143-701, Korea

Full list of author information is available at the end of the article
}

(c) The Author(s). 2020 Open Access This article is distributed under the terms of the Creative Commons Attribution 4.0 International License (http://creativecommons.org/licenses/by/4.0/), which permits unrestricted use, distribution, and reproduction in any medium, provided you give appropriate credit to the original author(s) and the source, provide a link to the Creative Commons license, and indicate if changes were made. The Creative Commons Public Domain Dedication waiver (http://creativecommons.org/publicdomain/zero/1.0/) applies to the data made available in this article, unless otherwise stated. 


\section{Background}

Plants have traditionally been used as an important source of pharmacologically active compounds that maintain human health. Notably, plants have been a major source of numerous antioxidant compounds essential in medicines, cosmetics, and the food industry [1]. In particular, the antioxidant metabolites in fruits and plant extracts have a range of health benefits such as maintaining cardiovascular health and cancer prevention, among many others [2, 3]. In herbal cosmetics, tyrosinase inhibitory compounds from plants are used as anti-melanogenic agents [4]. Tyrosinase, a copper-containing monooxygenase enzyme, is found widely in nature, including in plants, fungi, and animals. It catalyzes the hydroxylation of tyrosine to LDOPA (L-3,4-dihydroxyphenylalanine), which is subsequently oxidized to L-dopaquinone. This is then autopolymerized to form melanin pigments [5]. Hence, natural inhibitors of tyrosinase are considered vital for treating dermatological hyper-pigmentation caused by overproduction of melanin $[6,7]$.

Ushering into the metabolomics era, mass spectrometry (MS)- based metabolite characterization has evolved as an adept methodology to discern chemotaxonomy, metabolic pathways, and phytochemical characterization, complementing the omics-cascade alongside genomics and proteomics [8]. Metabolomics enables unbiased, highthroughput screening and characterization of the metabolite gamut in biological sample extracts through chromatographic separation, high resolution MS, and enhanced detection sensitivity [9-11]. However, the subsequent functional annotation of the identified metabolites often seems difficult, owing to the different titers of metabolic repertoire influencing biological phenotypes. Hence, neoteric multi-parallel approaches need to be explored for the expeditious screening and characterization of functional metabolites in diverse plant samples $[12,13]$.

It has been observed that plants of the same family usually synthesize compounds of similar classes, owing to the presence of similar biosynthetic pathways and regulatory enzymes [14]. Previously, discriminant metabolites, including the flavonoid contents in plant extracts, have been reported for varying taxonomic orders. However, the spatial metabolic disparity between different plant parts (leaves and stems) has not been deconstructed comprehensively $[8,15]$. In general, the chemical composition of different plant parts is largely influenced by genetic factors, nutritional status, and geo-climatic conditions [16]. Moreover, the varying distributions of functional metabolites including leaves, stems, and flowers, necessitating the need to delineate plant chemical diversity across the phylogenies as well spatial components [17-19].

Herein, a multi-parallel metabolomic-cum-bioassayguided approach toward the metabolomic characterization of different biosystematic groups is proposed for the three different plant families (Asteraceae, Fabaceae, and Rosaceae) across their spatial parts (leaf and stem). We employed untargeted mass spectrometry (MS)-based metabolomics coupled with biochemical phenotype analyses toward the comprehensive characterization of significantly discriminant metabolites contributing to the spatial and phylogenic chemodiversity among different plant species.

\section{Results \\ Metabolite profiling across spatial components (leaves and stems) and families (Asteraceae, Fabaceae, and Rosaceae)}

Herein, we examined the 2-D metabolomic profiles across the spatial components of 51 plant species belonging to three different families (Asteraceae, Fabaceae, and Rosaceae) that are widespread on the Korean peninsula. The subtle metabolic disparity among the plant samples was evaluated using GC-TOF-MS and UHPLCQ-Orbitrap-MS, followed by multivariate statistical analysis of the corresponding datasets. The principal components analysis (PCA) score plot based on the GCTOF-MS data displayed a clustered pattern for the 51 samples, segregating them into three distinct groups according to the corresponding plant families across PC 1 (8.2\%) and PC 2 (4.7\%), while their spatial metabolic disparity was evident along PC 2 (Fig. 1a). Further, the PCA based on the UHPLC-Q-Orbitrap-MS datasets indicated the marked variance across corresponding plant families and plant parts, along PC 1 (3.8\%) and PC 2 (3.3\%; Fig. 1c), respectively. The significantly discriminant metabolites between the spatial components that potentially contributed to the observed chemotaxonomic variance among the different plant species were statistically selected at variable importance in the projection (VIP) $>0.7$ or $p<0.05$, based on the PLS-DA model (Fig. $1 \mathrm{~b}, \mathrm{~d})$. Altogether, 64 metabolites including 41 primary metabolites and 23 secondary metabolites were identified as significantly discriminant. The primary metabolites were identified using standard compounds, and their spectrometric details are provided as Additional file 1: Table S1. Further, the secondary metabolites were characterized based on their UV-absorbance, masses $(\mathrm{m} / \mathrm{z})$, mass fragmentation patterns, and elemental composition (Additional file 2: Table S2). We comprehensively established the putative identities of these compounds using the published methodologies [20]. The metabolites characterized using the above described parameters were comparatively matched with online databases, standards, and in-house library, and crossconfirmed using the published literature at last. Intriguingly, the primary metabolite heterogeneity was mainly evident for spatial (leaf and stem) components, while the secondary metabolites were more diversified both spatially and across the plant families. 


\section{(A) PCA}

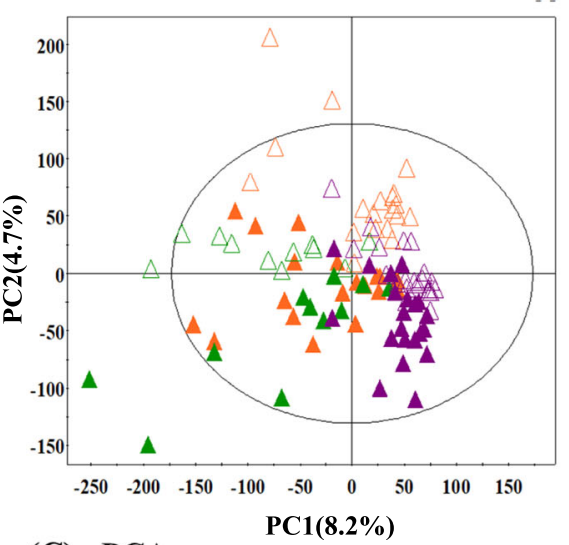

(C) PCA

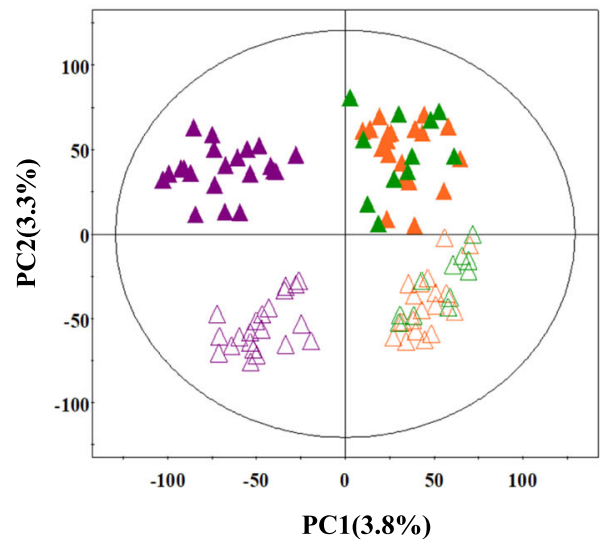

(B) PLS-DA

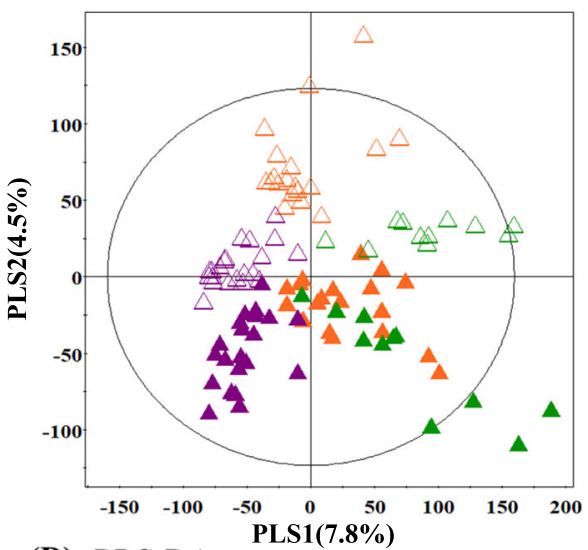

(D) PLS-DA

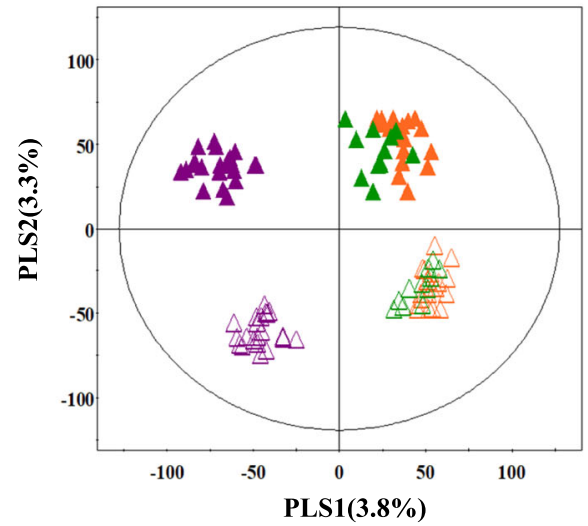

Fig. 1 PCA and PLS-DA score plots derived from the (a, b) GC-TOF-MS dataset and (c, $\mathbf{d})$ the UHPLC-Q-Orbitrap-MS dataset for leaves and stems of 51 indigenous plant species. ( $\mathbf{\Delta}$; Leaf, $\Delta ;$ Stem, Orange, Asteraceae; Green, Fabaceae; violet, Rosaceae)

Relative metabolite abundance in plant samples across spatial components and families

The metabolic pathways involved in the biosynthesis of significantly discriminant primary and secondary metabolites were mapped, and their relative abundances in the corresponding plant samples were indicated (Fig. 2). Considering the primary metabolite disparity among the three families, relatively higher levels of organic acids (caffeic acid, lactic acid, succinic acid, shikimic acid, and fumaric acid) were observed in Asteraceae extracts. On the contrary, amino acids (phenylalanine, tyrosine, GABA, serine, arginine, and aspartic acid) and fatty acids (stearic acid, oleic acid, palmitic acid, and oleamide) were abundant in Fabaceae extracts, while some metabolites including adonitol, sorbitol, and ferulic acid were detected at relatively higher levels in Rosaceae family extracts. In the case of secondary metabolites, the relative abundances of kaempferol glucoside and kaempferol-3-O- $\beta$-rutinoside were highest in Asteraceae extracts among the three families. On the contrary, genistein, naringenin, and isoorientin were most abundant in Fabaceae, whereas the relative levels of aceroside VIII, isoquercetin, quercetin-3-O-
glucosyl-6-O-pentoside, ellagic acid rhamnoside, pinocembrin, epicatechin, and catechin were detected highest in Rosaceae samples.

\section{Bioactivity correlations for the significantly discriminant metabolites}

The bioactivity of phytochemical extracts is mainly due to the diverse composition of secondary metabolites fulfilling multiple ecological roles among plant species. The spatial distributions of these compounds among varying phylogenic groups and plant components are remarkably discriminant. In this study, 102 (leaf and stem) sample extracts from 51 plant species were investigated for associated bioactivities using DPPH radical scavenging assays, total phenol content, total flavonoid content, and tyrosinase inhibitory assays (Fig. 3). The results of the bioactivity assays for the 51 species (Family: Asteraceae, Fabaceae, and Rosaceae) were estimated across plant families as well as their spatial components (leaf and stem). The average DPPH antioxidant activity was observed in the following order from highest to lowest; Rosaceae stems > Rosaceae leaves > Fabaceae leaves > 


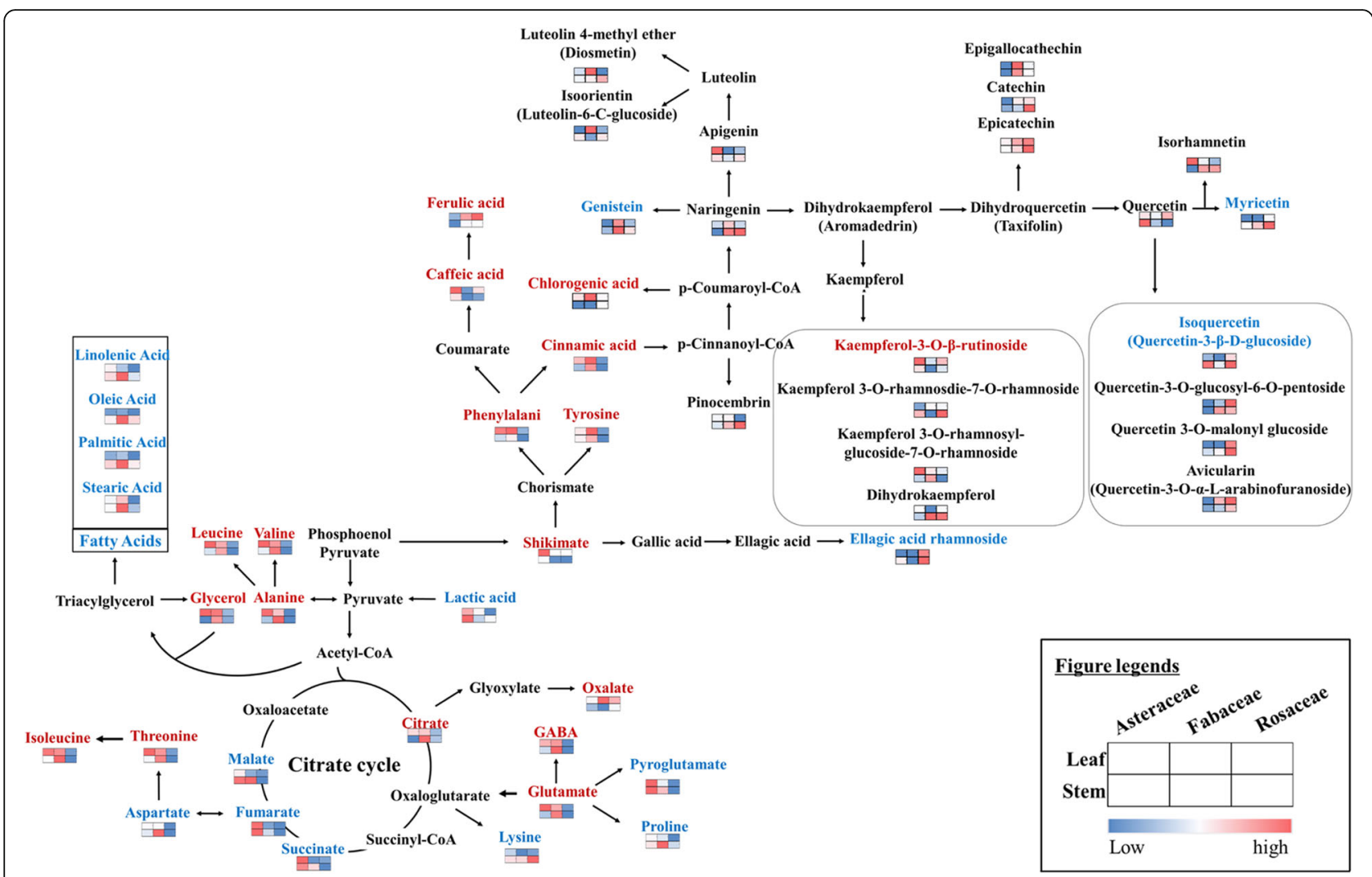

Fig. 2 Schematic diagram representing the relative contents of the significantly discriminant metabolites in their corresponding biosynthetic pathways across 51 plant species and across plant spatial parts. The modified pathway was adapted from the KEGG database (http://www. genome.jp/kegg/). The heatmap represents the mean value of the peak area for the discriminant metabolites detected across spatial parts (leaves and stems) for the three plant families (Asteraceae, Fabaceae, and Rosaceae). The discriminant metabolites among leaf and stem extracts are indicated in red and blue fonts, respectively. The colors of the heat map for metabolite levels represent their average fold-change values

\section{(A) DPPH}

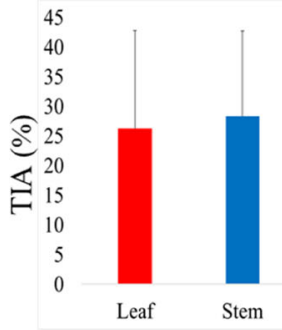

(B) Total phenol contents

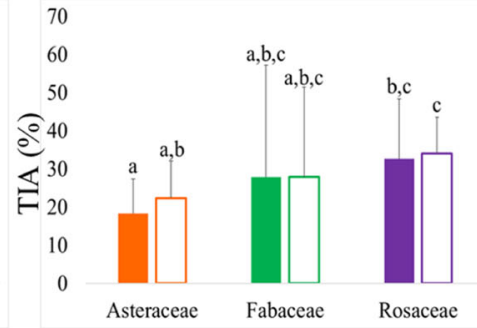

\section{(C) Total flavonoid contents}
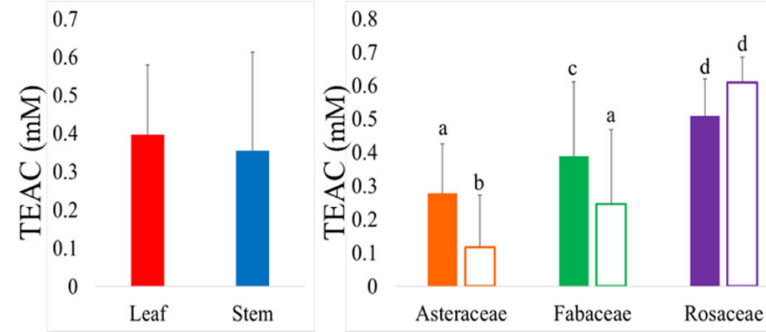
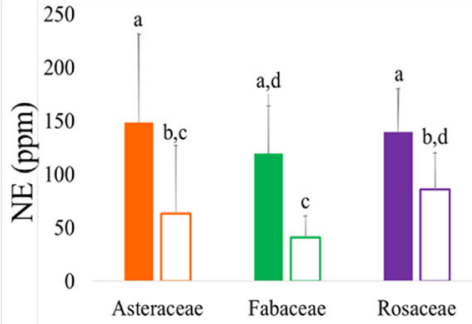

\section{(D) Tyrosinase inhibition activity}

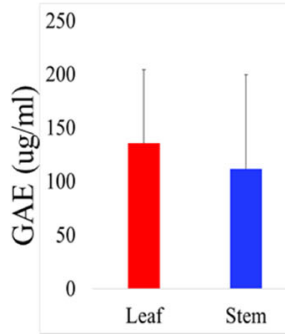

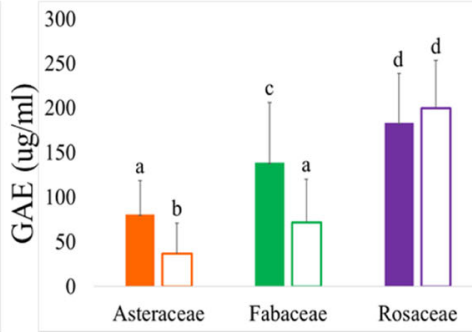

Fig. 3 Bioactivities of leaf and stem extracts from 51 indigenous plant species (a) Antioxidant activity using DPPH radical scavenging assays, (b) total phenol contents, (c) total flavonoid contents, and (d) tyrosinase inhibition activity 
Asteraceae leaves $>$ Fabaceae stems $>$ Asteraceae stems (Fig. 3a). Similarly, the average total phenol contents were observed in the following order; Rosaceae stems > Rosaceae leaves $>$ Fabaceae leaves $>$ Asteraceae leaves $>$ Fabaceae stems $>$ Asteraceae stems (Fig. 3b). On the contrary, average values for the total flavonoid content varied in the following order: Asteraceae leaves $>$ Rosaceae leaves $>$ Fabaceae leaves $>$ Rosaceae stems $>$ Asteraceae stems $>$ Fabaceae stems (Fig. 3d). In general, higher antioxidant activity was observed in leaf extracts compared to stem samples, except for the Rosaceae family. On the contrary, average tyrosinase inhibitory activity was significantly higher in stem samples than in leaf samples, irrespective of the plant family.

Pearson's correlation analysis tentatively identified compounds that contributed maximally to the observed biological activities of the plant extracts. The correlation network was evaluated for variables with a Pearson correlation value $>0.3$ (Fig. 4). Intriguingly, aceroside VIII, ellagic acid rhamnose, catechin, epicatechin, mannitol, quercetin3-O-malonylglucoside, and quercetin-3-O-glucosyl-6-Opentoside showed strong positive correlations with both total phenol content (TPC) and DPPH antioxidant activity.

\section{Discussion}

We applied untargeted metabolomics coupled with bioactivity assays to evaluate the two dimensional (2D) metabolomic diversity across 51 species belonging to three major plant families and across their spatial plant parts (leaves and stems). The chemical composition of the different plant parts is affected by multiple factors including genetics, metabolic factors, and geo-climatic conditions [14]. Previously, we have highlighted the importance of mass spectrometry (MS)-based metabolomics to identify the chemotaxonomic profiles of different plant samples across various genera and families [8]. The untargeted metabolomics followed by multivariate analyses indicated that metabolite profiles varied significantly for the plant samples across different phylogenies and spatial parts irrespective of the variations in geographical location or in the period of sample collection (2011-2015).

The levels of amino acids are relatively higher in Fabaceae species compared to Cornaceae and Rosaceae [15]. Further, genistein, an important isoflavone is well reported from soybean and other edible Fabaceae species, along with naringenin and isoorientin [21]. In congruence, we observed that amino acids and isoflavones were abundant in Fabaceae, while the kaempferol and quercetin derivatives were relatively higher in Asteraceae and Rosaceae family extracts, respectively. Reportedly, the kaempferol glycosides are the main polyphenolic compounds in Asteraceae family plants [22]. Whereas, quercetin glycosides are the typical flavonol glycoside in the Rosaceae family, along with flavan-3-ol (catechin and epicatechin) and polyphenol compounds, including ellagic acid [23].

Considering the spatial disparity in metabolites between leaf and stem components, the levels of branch chain amino acids (BCAA) (isoleucine, leucine, valine), aromatic amino acids (AAA) (phenylalanine, tyrosine), phenylpropanoids (cinnamic acid, caffeic acid, ferulic acid, shikimic acid), sugar alcohols (xylitol, myo-inositol, meso-erythritol), and glycerol derivatives (glycerol, glyceryl-glyceryl) were relatively higher in leaves than in stem extracts. Branch chain amino acids cannot be synthesized by animals; however, plants can synthesize these amino acids de novo and thus serve as an important source of these compounds in the human diet [24]. In plants, isoleucine, leucine, and

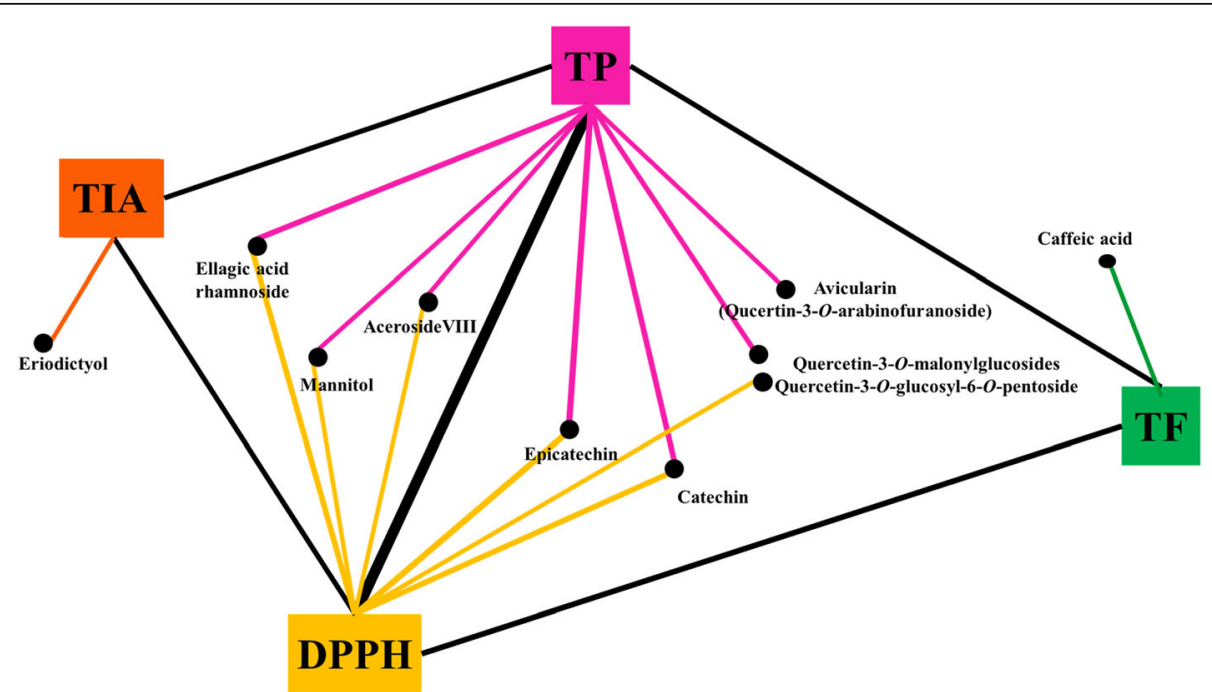

Fig. 4 Correlation networks between the metabolites and bioactivity assays (DPPH, TP, TF, and tyrosinase inhibition activity). The metabolites were selected based on a Pearson's correlation value $(r)>0.3$ 
valine share common BCAA-hydrolyzing enzymes in their biosynthetic pathways. Accumulation of free amino acids plays an important role in plant stress tolerance, and these can act as osmolytes under certain abiotic stress conditions [25]. Similarly, phenylpropanoids are key components with antioxidant functions that ameliorate high intensity lightstress mediated damage in leaves [26]. On the contrary, the levels of fatty acids (oleic acid, stearic acid, palmitic acid, and linolenic acid) and amino acids (proline, lysine, and arginine) were relatively higher in leaves compared to stem extracts. Oleic and linolenic acid derivatives partially regulate plant development, seed colonization, and defense responses to pathogens through various mechanisms [27, 28]. Notably, the relative abundances of chlorogenic acid and the majority of flavonoids (dihydrokaempferol, quercetin, quercetin derivatives, and myricetin derivatives) were higher in leaves, while kaempferol glucoside and pinocembrin were more abundant in stems. The higher abundance of flavonoids in leaves might be attributed to their local biosynthesis as well as their active translocation from other plant organs at different stages of development [29].

Generally, similar antioxidant activity levels were observed in the same genus groups, but the species belonging to the genera Alnus displayed significantly different antioxidant levels. These results suggest that the differences in chemical compositions among species belonging to the same genus may be expressed in terms of their varying chemotaxonomy and associated bioactivities. In the present study, multivariate analyses indicated distinct metabolite profiles for plant extracts according to different plant families and spatial parts. Hence, the chemotaxonomic hierarchy of plants depends on their biosynthetic relatedness to synthesize corresponding metabolite pools [30].

We observed that aceroside VIII, catechin, and quercetin derivatives were positively correlated with DPPH antioxidant phenotypes, whereas eriodictyol was associated with tyrosinase inhibition effects. According to recent studies, aceroside VIII is an acerogenin derivative with significant antioxidant activities [31]. Ellagic acid is structurally a phenol antioxidant that exhibits significant free radical scavenging activity. It also promotes the activity of three antioxidant enzymes, namely superoxide dismutase (SOD), catalase (CAT), and glutathione peroxidase (GPX), which are altered under various physiological states involving free radical attack [32]. The roles of mannitol as an osmo-protectant as well as free radical scavenger that influences the activities of antioxidant enzymes including SOD, CAT, glutathione reductase (GR), peroxidase (POX), and ascorbate peroxidase (APX) have also been established [33]. Previously, Iacopini et al. described catechin, epicatechin, and quercetin as phenolic compounds that can independently or synergistically exhibit DPPH radical activities [34]. However, in the present study, eriodictyol and caffeic acid were linked to tyrosinase inhibition and total flavonoid content assays, respectively. Notably, eriodictyol is a flavonoid that can inhibit melanogenesis [35].

\section{Conclusion}

The present study construed the chemometric profiles of 51 plant samples across phylogenic groups and spatial parts, correlating their untargeted metabolite profiles with corresponding bioactivity phenotypes. Notwithstanding the effects of varying harvest time and regions, we observed distinct metabolomic profiles with higher antioxidant and tyrosinase inhibitory activities for leaf and stem extracts, respectively, across different families. Correlation analyses indicated that several metabolites either independently or synergistically affected the antioxidant phenotypes in plant extracts. Considering the subtle mechanisms, the observed chemical diversity for varying plant samples provided an insight of the specialized metabolic pathways which may have affected the phenotypic variance. Further, the holistic metabolite repertoire and associated bioactivities reported in the study can supplement the existing compendium of plant metabolomics data.

\section{Methods}

\section{Chemicals and reagents}

HPLC-grade water, ethanol, methanol, and acetonitrile were purchased from Fisher Scientific (Pittsburgh, PA, USA). Formic acid, N-methyl-N-(trimethylsilyl) trifluoroacetamide (MSTFA), Methoxyamine hydrochloride, pyridine, 2,2-diphenyl-1-pricrylhydrazyl (DPPH), sodium hydroxide, diethylene glycol, Folin-Ciocalteu's phenol reagent, and sodium carbonate were purchased from Sigma Aldrich (St. Louis, MO, USA).

\section{Plant materials}

We procure five different plant samples for each of the 51 species belonging to three different plant families from the National Institute of Biological Resources (NIBR, Incheon, Korea). The plant samples were dried under shade, pooled, and ground into fine homogeneous powders using the Mixer Mill. Information regarding the plants used in this study is listed in Table 1.

\section{Sample extract preparation}

Approximately $1 \mathrm{~g}$ of sample powder was extracted using $10 \mathrm{~mL}$ of $80 \%$ methanol following continuous shaking at $200 \mathrm{rpm}$ for $24 \mathrm{~h}$. The resulting mixture was cold centrifuged $\left(4^{\circ} \mathrm{C}\right)$ at $2800 \times g$ for $15 \mathrm{~min}$ (Hettich Zentrifugen, Universal 320), and the supernatant was filtered using a $0.2 \mu \mathrm{m}$ syringe. The supernatant was dried under a speed-vacuum concentrator (Modulspin 31, Biotron, Korea), and was resuspended in $80 \%$ methanol at an 
Table 1 Basic information about the 51 species of plant samples

\begin{tabular}{|c|c|c|c|c|}
\hline No. & Species & Family & Collection date & Collection Site \\
\hline 1 & Hemistepta lyrata & Asteraceae & 2015-05-27 & Hyeon-ri, Girin-myeon, Inje-gun, Gangwon-do \\
\hline 2 & Breea segeta & Asteraceae & 2015-06-08 & Oksan-ri, Hojeo-myeon, Wonju-si, Gangwon-do \\
\hline 3 & Aster incisus & Asteraceae & 2015-08-11 & Myeong-wol-ri, Sanae-myeon, Hwacheon-gun, Gangwon-do \\
\hline 4 & Syneilesis palmata & Asteraceae & 2015-08-13 & Hantan-ri, Mitan-myeon, Pyeongchang-gun, Gangwon-do \\
\hline 5 & Sigesbeckia glabrescens & Asteraceae & 2015-08-29 & Udu-ri, Dolsan-eup, Yeosu-si, Jeollanam-do \\
\hline 6 & Artemisia montana & Asteraceae & 2015-09-02 & Namseo-ri, Seo-myeon, Ulleung-gun, Gyeongsangbuk-do \\
\hline 7 & Sigesbeckia pubescens & Asteraceae & 2015-09-04 & Gujeol-ri, Yeoryang-myeon, Jeongseon-gun, Gangwon-do \\
\hline 8 & Synurus deltoides & Asteraceae & 2015-09-05 & Hyeol-dong, Taebaek-si, Gangwon-do \\
\hline 9 & Cirsium japonicum & Asteraceae & 2015-09-07 & Daepo-dong, Seogwipo-si, Jeju special self-governing province \\
\hline 10 & Sonchus asper & Asteraceae & 2015-09-08 & Bomok-dong, Seogwipo-si, Jeju special self-governing province \\
\hline 11 & Atractylode ovata & Asteraceae & 2015-09-12 & Baegil-ri, Gwayeok-myeon, Goheung-gun, Jeollanam-do \\
\hline 12 & Dendranthema boreale & Asteraceae & 2015-10-06 & Sasong-ri, Baekgok-myeon, Jincheon-gun, Chungcheongbuk-do \\
\hline 13 & Helianthus tuberosus & Asteraceae & 2014-08-12 & Jiro-ri, Byeongyeong-myeon, Gangjin-gun, Jeollanam-do \\
\hline 14 & Conyza canadensis & Asteraceae & 2014-08-14 & Sangdodae-ri, Sangchon-myeon, Yeongdong-gun, Chungcheongbuk-do \\
\hline 15 & Artemisia capillaris & Asteraceae & 2014-08-21 & Nadae-ri, Yaro-myeon, Hapcheon-gun, Gyeongsangnam-do \\
\hline 16 & Saussurea pulchella & Asteraceae & 2014-08-30 & Gohan-ri, Gohan-eup, Jeongseon-gun, Gangwon-do \\
\hline 17 & Erigeron annuus & Asteraceae & 2014-08-05 & Dongmak-ri, Yeoncheon-eup, Yeoncheon-gun, Gyeonggi-do \\
\hline 18 & Bidens bipinnata & Asteraceae & 2014-08-19 & Dongmak-ri, Yeoncheon-eup, Yeoncheon-gun, Gyeonggi-do \\
\hline 19 & Lactuca indica & Asteraceae & 2014-08-24 & Gomo-ri, Soheul-eup, Pocheon-si, Gyeonggi-do \\
\hline 20 & Vicia amoena & Fabaceae & 2015-08-11 & Guun-ri, Sangseo-myeon, Hwacheon-gun, Gangwon-do \\
\hline 21 & Chamaecrista nomame & Fabaceae & 2015-08-18 & Masan-dong, Gimpo-si, Gyeonggi-do \\
\hline 22 & Robinia pseudoacacia & Fabaceae & $2014-10-23$ & Sin-ri, Goryeong-eup, Goryeong-gun, Gyeongsangbuk-do \\
\hline 23 & Albizia julibrissin & Fabaceae & 2014-08-06 & Daechi-ri, Daechi-myeon, Cheongyang-gun, Chungcheongnam-do \\
\hline 24 & Sophora flavescens & Fabaceae & 2014-08-18 & Hanggok-ri, Gunbuk-myeon, Okcheon-gun, Chungcheongbuk-do \\
\hline 25 & Lespedeza cuneata & Fabaceae & 2014-08-22 & Geogi-ri, Jusang-myeon, Geochang-gun, Gyeongsangnam-do \\
\hline 26 & Desmodium caudatum & Fabaceae & 2014-08-24 & Seonheul-ri, Jocheon-eup, Jeju-si, Jeju special self-governing province \\
\hline 27 & Pueraria lobata & Fabaceae & 2014-08-04 & Mamyeong-ri, Naechon-myeon, Pocheon-si, Gyeonggi-do \\
\hline 28 & Lespedeza maximowiczii & Fabaceae & 2011-08-03 & Giri, Gajo-myeon, Geochang-gun, Gyeongsangnam-do \\
\hline 29 & Lespedeza bicolor & Fabaceae & 2011-08-04 & Ongdong-myen, Jeongeup-si, Jeollabuk-do \\
\hline 30 & Lespedeza cyrtobotrya & Fabaceae & 2011-07-02 & Wang-dong, Gwangsan-gu, Gwanju \\
\hline 31 & Rubus corchorifolius & Rosaceae & 2015-04-22 & Cheongyong-ri, Gogeum-myeon, Wando-gun, Jeollanam-do \\
\hline 32 & Spiraea prunifolia & Rosaceae & 2015-04-29 & Jindong-ri, Girin-myeon, Inje-gun, Gangwon-do \\
\hline 33 & Rubus parvifolius & Rosaceae & 2015-06-02 & Gwansan-ri, Yaksan-myeon, Wando-gun, Jeollanam-do \\
\hline 34 & Rosa multiflora & Rosaceae & 2015-06-03 & Gwansan-ri, Yaksan-myeon, Wando-gun, Jeollanam-do \\
\hline 35 & Prunus sargentii & Rosaceae & 2015-06-08 & Oe-ri, Yeongheung-myeon, Ongjin-gun, Incheon \\
\hline 36 & Prunus davidiana & Rosaceae & 2015-06-30 & Sanggeol-ri, Dong-myeon, Chuncheon-si, Gangwon-do \\
\hline 37 & Sanguisorba tenuifolia & Rosaceae & 2015-09-17 & Donggo-ri, Sinji-myeon, Wando-gun, Jeollanam-do \\
\hline 38 & Sorbus commixta & Rosaceae & 2014-07-16 & Jeodong-ri, Ulleung-eup, Ulleung-gun, Gyeongsangbuk-do \\
\hline 39 & Prunus armeniaca & Rosaceae & 2014-07-20 & Ojeong-dong, Daedeok-gu, Daejeon \\
\hline 40 & Pyrus ussuriensis & Rosaceae & 2014-08-01 & Icheon-ri, Sangbuk-myeon, Ulju-gun, Ulsan \\
\hline 41 & Prunus yedoensis & Rosaceae & 2014-08-07 & Janghyeon-ri, Cheongna-myeon, Boryeong-si, Chungcheongnam-do \\
\hline 42 & Spiraea salicifolia & Rosaceae & 2014-08-08 & Ungyo-ri, Bangnim-myeon, Pyeongchang-gun, Gangwon-do \\
\hline 43 & Chaenomeles sinensis & Rosaceae & 2014-08-10 & Ojeong-dong, Daedeok-gu, Daejeon \\
\hline 44 & Eriobotrya japonica & Rosaceae & 2014-08-13 & Jiro-ri, Byeongyeong-myeon, Gangjin-gun, Jeollanam-do \\
\hline
\end{tabular}


Table 1 Basic information about the 51 species of plant samples (Continued)

\begin{tabular}{lllll}
\hline No. & Species & Family & Collection date & Collection Site \\
\hline 45 & Rubus coreanus & Rosaceae & $2014-08-14$ & Sogye-ri, Hwanggan-myeon, Yeongdong-gun, Chungcheongbuk-do \\
46 & Rubus crataegifolius & Rosaceae & $2014-08-21$ & Nadae-ri, Yaro-myeon, Hapcheon-gun, Gyeongsangnam-do \\
47 & Rubus phoenicolasius & Rosaceae & $2014-08-21$ & Nadae-ri, Yaro-myeon, Hapcheon-gun, Gyeongsangnam-do \\
48 & Pourthiaea villosa & Rosaceae & $2014-08-24$ & Seonheul-ri, Jocheon-eup, Jeju-si, Jeju special self-governing province \\
49 & Prunus maackii & Rosaceae & $2014-08-30$ & Gurae-ri, Sangdong-eup, Yeongwol-gun, Gangwon-do \\
50 & Prunus padus & Rosaceae & $2014-05-22$ & Gohan-ri, Gohan-eup, Jeongseon-gun, Gangwon-do \\
51 & Prunus $s p$. & Rosaceae & $2014-08-08$ & Gomo-ri, Soheul-eup, Pocheon-si, Gyeonggi-do \\
\hline
\end{tabular}

appropriate concentration. This suspension was then analyzed by UHPLC-Q-Orbitrap-MS to detect secondary metabolites. For GC-TOF-MS analysis, extracts were oximated using methoxyamine hydrochloride $(20 \mathrm{mg}$ $\mathrm{mL}^{-1}$ ) in pyridine at $30{ }^{\circ} \mathrm{C}$ for $90 \mathrm{~min}$. Then, the oximated samples were silylated with MSTFA at $37^{\circ} \mathrm{C}$ for $30 \mathrm{~min}$. All MS analyses were conducted with three analytical replications.

\section{Bioactivity assays DPPH assay}

Measurement of antioxidant activity was carried out with DPPH assays, following the methodology originally proposed by Villano et al. [36], with some modifications. In brief, the DPPH $(200 \mu \mathrm{mol})$ reagent was dissolved in ethanol and maintained for $20 \mathrm{~min}$ at $60-70{ }^{\circ} \mathrm{C}$ until the solution absorbance reached $1.0 \pm 0.02$ at $515 \mathrm{~nm}$, as measured by a spectrophotometer (Thermo Electron, Spectronic Genesys 6, Madison, WI, USA). The resulting solution was kept stable for the next $16 \mathrm{~h}$ and stored at $4{ }^{\circ} \mathrm{C}$. The assays were performed by adding $180 \mu \mathrm{L}$ of the DPPH solution to the plant sample extracts $(20 \mu \mathrm{L}, 1$ $\mathrm{mg} \mathrm{mL}^{-1}$ ), and the resulting mixture was incubated for $20 \mathrm{~min}$ at $37^{\circ} \mathrm{C}$ in the dark. The reaction absorbance was measured at $515 \mathrm{~nm}$. Results were expressed as Trolox equivalent activity concentrations (mM), and as the mean value of the three analytical replicates.

\section{Total phenol content}

Total phenol content assays were performed in two steps. First, the reaction mixture, containing $20 \mu \mathrm{L}$ of plant sample extract in $80 \%$ methanol $\left(1 \mathrm{mg} \mathrm{mL}^{-1}\right)$ and $100 \mu \mathrm{L}$ of $0.2 \mathrm{~N}$ Folin-Ciocalteu's phenol reagent, was incubated for $5 \mathrm{~min}$ in the dark. Then, $80 \mu \mathrm{L}$ of $7.5 \%$ $\mathrm{Na}_{2} \mathrm{CO}^{3}$ was added, and the resulting reaction mixture was incubated for $60 \mathrm{~min}$. Finally, absorbance was measured at $750 \mathrm{~nm}$. Assay results were expressed in terms of gallic acid equivalent of the activity $\left(\mu \mathrm{g} \mathrm{mL} L^{-1}\right)$, and as the mean value of three analytical replicates.

\section{Total flavonoid content (stock 1000 ppm)}

For total flavonoid content assays, reaction mixtures contained $20 \mu \mathrm{L}$ of plant sample extract in $80 \%$ methanol $\left(1 \mathrm{mg} \mathrm{mL}^{-1}\right), 20 \mu \mathrm{L}$ of $0.1 \mathrm{~N} \mathrm{NaOH}$, and $160 \mu \mathrm{L}$ of $90 \%$ diethylene glycol. The reaction mixture was incubated for $60 \mathrm{~min}$ and the resulting absorbance was recorded at $405 \mathrm{~nm}$. Results were expressed as naringin equivalent activity concentrations $\left(\mu \mathrm{g} \mathrm{mL}^{-1}\right)$. The data were presented as the mean of three analytical replicates.

\section{Tyrosinase inhibitory activity}

Mushroom tyrosinase inhibitory activity was determined using the following method. A reaction mixture was prepared with $125 \mu \mathrm{L}$ of $0.1 \mathrm{M}$ sodium phosphate buffer ( $\mathrm{pH} 6.5$ ), $5 \mu \mathrm{L}$ of plant sample extract in $80 \%$ methanol (10 $\mathrm{mg} \mathrm{mL}^{-1}$ ), $30 \mu \mathrm{L}$ of mushroom tyrosinase (1000 unit $\left.\mathrm{mL}^{-1}\right)$, and $40 \mu \mathrm{L}$ of $1.5 \mathrm{mM} \mathrm{L}$-tyrosine, and was added to 96 -well plates. The reaction mixture was incubated at $37^{\circ} \mathrm{C}$ for $20 \mathrm{~min}$ and absorbance was measured at 490 $\mathrm{nm}$. The data were presented as the mean value of three analytical replicates.

\section{Mass spectrometry (MS) analysis GC-TOF-MS analysis}

An Agilent 7890A gas chromatography (GC) system equipped with an Agilent 7693 autosampler coupled to a Pegasus Time-of-Flight Mass Spectrometer (TOF-MS) detector (Leco Corporation, St. Joseph, MI, USA) was used for GC-TOF-MS analyses as described by Lee et al. [13].

\section{UHPLC-Q-Orbitrap-MS analysis}

Samples were analyzed using a Q-Exactive Orbitrap MS equipped with a heated electrospray ionization source (Thermo Fischer Scientific, CA, USA), which consisted of a DIONEX UltiMate 3000 UHPLC system (Ultimate 3000 RS pump, Ultimate 3000 RS column compartment, and Ultimate 3000 RS autosampler; Dionex Corporation, CA, USA). Samples were separated on a hypersil gold C18 selectivity LC column (i.d., $1.9 \mu \mathrm{m}, 50 \times 2.1 \mathrm{~mm}$; Thermo Fisher scientific) at a column oven temperature 
of $25^{\circ} \mathrm{C}$. The mobile phases consisted of $0.1 \%$ formic acid in water (B) and in acetonitrile (C), and the compositions of the gradient flows were the same. The gradient was gradually increased from $0 \%$ solvent C to $100 \%$ solvent $C$ over $20 \mathrm{~min}$, and was maintained for a further 2 min. The flow rate was $0.3 \mathrm{~mL} \mathrm{~min}^{-1}$ and the injection volume was $10 \mu \mathrm{L}$. Mass spectra were obtained using electrospray ionization in negative and full scan modes within a range of $\mathrm{m} / \mathrm{z} 100-1000$. The operating parameters were as follows: spray needle voltage, $\pm 3.3 \mathrm{kV}$; capillary temperature, $320^{\circ} \mathrm{C}$; probe heater temperature, $300^{\circ} \mathrm{C}$; stacked ring ion guide (S-lens) radio frequency (RF) level, 60\%; resolution (full-width at half-maximum; FWHM), 35,000.

\section{Ultra performance liquid chromatography-quadrupole-time} of flight mass spectrometry (UPLC-Q-TOF-MS) analysis UPLC-Q-TOF-MS analyses were performed using a Waters Micromass Q-TOF Premier as described by Son et al. [15]. The mobile phase consisted of $0.1 \% \mathrm{v} / \mathrm{v}$ formic acid in water (A) and in acetonitrile (B). The solvent gradient system consisted of the following: $B$ was increased from 5 to $100 \%(\mathrm{v} / \mathrm{v})$ over $11 \mathrm{~min}$ and was maintained at $100 \%$ for $12 \mathrm{~min}$. Then, B was decreased to $5 \%$ in $0.01 \mathrm{~min}$, and was maintained at this level up to 13 min. The sample injection volume was $5 \mu \mathrm{L}$ and the flow rate was maintained at $0.3 \mathrm{~mL} \mathrm{~min}^{-1}$.

\section{Data processing and multivariate analysis}

GC-TOF-MS data files were converted to CDF format using ChromaTOF software v4.44 (Leco Co., CA, USA). LC-MS data (".raw) were converted to netCDF (".cdf) format using Xcalibur (version 2.2; Thermo Fischer Scientific, CA, USA). After conversion, the CDF format data were processed using the metAlign software package, and SIMCA-P + 12.0 (Umetrics, Umea, Sweden) for the principal component analysis (PCA) and partial least squares discriminant analysis (PLS-DA) modeling as described by Lee et al. [13]. The significantly different ( $p$ value $<0.05)$ metabolites contributing to the statistical variance among plant species were tested using one-way ANOVA on STATISCA (version 7.0, StaSoft Inc., Tulsa, OK, USA).

In the antioxidant and tyrosinase inhibition activity tests, differences were discerned by t-tests using PASW Statistics 18 (SPSS Inc., Chicago, IL, USA). Pairwise correlations between metabolites and bioactivities (antioxidant activity and tyrosinase inhibition activity) were calculated by Pearson's correlation coefficient using PASW Statistics 18. The correlations between metabolites and antioxidant bioactivity were visualized using heat map representations made with MEV software 4.8 (multiple array viewer, http://www.tm4.org/).

\section{Supplementary information}

Supplementary information accompanies this paper at https://doi.org/10. 1186/s12870-019-2231-y.

Additional file 1. Tentatively identified primary metabolits in plants based on metabolite profiling with multivariate analysis.

Additional file 2. Tentatively identified secondary metabolits in plants based on metabolite profiling with multivariate analysis.

\begin{abstract}
Abbreviations
AAA: Aromatic amino acid; APX: Ascorbate peroxidase; BCAA: Branch chain amino acid; CAT: Catalase; DPPH: 2,2-diphenyl-1-pricrylhydrazyl; GC-TOFMS: Gas chromatography time-of-flight mass spectrometry; GPX: Glutathione peroxidase; GR: Glutathione reductase; HPLC: High-performance liquid chromatography; MS: Mass spectrometry; MSTFA: Methoxyamine hydrochloride, N-methyl-N-(trimethylsilyl) trifluoroacetamide; PCA: Principal component analysis; PLS-DA: Partial least squares discriminant analysis; POX: Peroxidase; SOD: Superoxide dismutase; UHPLC-Q-orbitrap-MS/ MS: Ultrahigh-performance liquid chromatography quadrupole orbitrap ion trap tandem mass spectrometry; VIP: Variable importance in the projection
\end{abstract}

\section{Acknowledgements}

Not Applicable.

\section{Authors' contributions}

CHL and SaL provided the concept and designed this research. JSL and SaL provided samples. SuL performed most of the experimental work and data analysis. SuL, D-GO, DS, JSL, SaL, and CHL conducted the data interpretation. SuL wrote the manuscript. All authors read and approved the final manuscript.

\section{Funding}

This work was supported by the Korea Institute of Planning and Evaluation for Technology in Food, Agriculture, Forestry (IPET) through the High Valueadded Food Technology Development Program, funded by the Ministry of Agriculture, Food and Rural Affairs (MAFRA) (grant number 318027-04-3HD030), by a grant from the National Institute of Biological Resources (NIBR) funded by the Ministry of Environment (MOE) of the Republic of Korea (NIBR201912102), and by WTU Joint Research Grants of Konkuk University in 2017. The funders had no role in the design of the study and collection, analysis, and interpretation of data and in writing the manuscript.

Availability of data and materials

The data sets supporting the results of this article are included within the article and its additional files.

Ethics approval and consent to participate

Not applicable.

Consent for publication

Not applicable.

Competing interests

The authors declare that they have no competing interests.

\section{Author details}

${ }^{1}$ Department of Bioscience and Biotechnology, Konkuk University, Seoul 143-701, Korea. ${ }^{2}$ National Institute of Biological Resources, Environmental Research Complex, Incheon 22755, Korea. ${ }^{3}$ Research Institute for Bioactive-Metabolome Network, Konkuk University, Seoul 05029, Korea.

Received: 9 July 2019 Accepted: 30 December 2019

Published online: 28 January 2020

\section{References}

1. Ribeiro AS, Estanqueiro M, Oliveira MB, Sousa Lobo JM. Main benefits and applicability of plant extracts in skin care products. Cosmetics. 2015;2:48-65.

2. Javanmardi J, Stushnoff C, Locke E, Vivanco JM. Antioxidant activity and total phenolic content of Iranian Ocimum accessions. Food Chem. 2003;83: 547-50. 
3. Pulido R, Bravo L, Saura-Calixto F. Antioxidant activity of dietary polyphenols as determined by a modified ferric reducing/antioxidant power assay. J Agric Food Chem. 2000;48:3396-402.

4. Kumar KS, Yang JC, Chu FH, Chang ST, Wang SY. Lucidone, a novel melanin inhibitor from the fruit of Lindera erythrocarpa Makino. Phytother Res. 2010; 24:1158-65.

5. Hwang JH, Lee BM. Inhibitory effects of plant extracts on tyrosinase, L-DOPA oxidation, and melanin synthesis. J Toxicol Environ Health Part A. 2007;70: 393-407.

6. Chen CY, Kuo PL, Chen YH, Huang JC, Ho ML, Lin RJ, et al. Tyrosinase inhibition, free radical scavenging, antimicroorganism and anticancer proliferation activities of Sapindus mukorossi extract. J Taiwan Inst Chem Eng. 2010:41:129-35.

7. Shiino M, Watanabe $\mathrm{Y}$, Umezawa K. Synthesis of N-substituted Nnitrosohydroxylamines as inhibitors of mushroom tyrosinase. Bioorg Med Chem. 2001;9:1233-40

8. Lee S, Oh DG, Lee S, Kim GR, Lee JS, Son YK, et al. Chemotaxonomic metabolite profiling of 62 indigenous plant species and its correlation with bioactivities. Molecules. 2015;20:19719-34.

9. Kang D, Son GH, Park HM, Kim J, Choi JN, Kim HY, et al. Culture conditiondependent metabolite profiling of Aspergillus fumigatus with antifungal activity. Fungal Biol. 2013;117:211-9.

10. Kim J, Choi JN, Choi JH, Cha YS, Muthaiya MJ, Lee CH. Effect of fermented soybean product (Cheonggukjang) intake on metabolic parameters in mice fed a high-fat diet. Mol Nutr Food Res. 2013;57:1886-91.

11. Lee S, Do SG, Kim SY, Kim J, Jin Y, Lee CH. Mass Spectrometry-Based Metabolite Profiling and Antioxidant Activity of Aloe vera (Aloe barbadensis Miller) in Different Growth Stages. J Agric Food Chem. 2012;60:11222-8.

12. Chen W, Gong L, Guo Z, Wang W, Zhang H, Liu X, Yu S, Xiong L, Luo J. A novel integrated method for large-scale detection, identification, and quantification of widely targeted metabolites: application in the study of rice metabolomics. Mol Plant. 2013:6:1769-80.

13. Lee $S$, Oh DG, Singh D, Lee HJ, KIM GR, Lee S, Lee JS, Lee CH. Untargeted Metabolomics Toward Systematic Characterization of Antioxidant Compounds in Betulaceae Family Plant Extracts. Metabolites. 2019;9:186.

14. Ntie-Kang F, Lifongo LL, Mbaze LMA, Ekwelle N, Owono LCO. Megnassan E. Cameroonian medicinal plants: a bioactivity versus ethnobotanical survey and chemotaxonomic classification. BMC Complement Altern Med. 2013;13:147.

15. Son SY, Kim NK, Lee S, Singh D, Kim GR, Lee JS, et al. Metabolite fingerprinting, pathway analyses, and bioactivity correlations for plant species belonging to the Cornaceae, Fabaceae, and Rosaceae families. Plant Cell Rep. 2016;35:917-31.

16. Dias MI, Sousa MJ, Alves RC, Ferreira IC. Exploring plant tissue culture to improve the production of phenolic compounds: A review. Ind Crop Prod. 2016;82:9-22.

17. del Baño MJ, Lorente J, Castillo J, Benavente-García O, del Río JA, Ortuño A, et al. Phenolic diterpenes, flavones, and rosmarinic acid distribution during the development of leaves, flowers, stems, and roots of Rosmarinus officinalis. Antioxidant activity. J Agric Food Chem. 2003;51:4247-53.

18. Bettaieb I, Bourgou S, Wannes WA, Hamrouni I, Limam F, Marzouk B. Essential oils, phenolics, and antioxidant activities of different parts of cumin (Cuminum cyminum L.). J Agric Food Chem. 2010:58:10410-8.

19. Shahhoseini R, Hosseini N, Ghorbanpour M. Study of essential oil content and composition of different parts of lemon verbena (Lippia citriodora) grown in Iran. J Essent Oil Bear Plants. 2014;17:120-5.

20. Lynn KS, Cheng ML, Chen YR, Hsu C, Chen A, Lih M, et al. Metabolite identification for mass spectrometry-based metabolomics using multiple types of correlated ion information. Anal Chem. 2015;87:2143-51.

21. Spinola V, Llorent-Martinez EJ, Gouveia-Figueira S, Castilho PC. Ulex europaeus: from noxious weed to source of valuable isoflavones and flavanones. Ind Crop Prod. 2016;90:9-27.

22. Mejía-Giraldo JC, Winkler R, Gallardo C, Sánchez-Zapata AM, Puertas-Mejía MA. Photoprotective potential of Baccharis antioquensis (Asteraceae) as natural sunscreen. Photochem Photobiol. 2016;92:742-52.

23. Määttä-Riihinen KR, Kamal-Eldin A, Törrönen AR, Identification and quantification of phenolic compounds in berries of Fragaria and Rubus species (family Rosaceae). J Agric Food Chem. 2004;52:6178-87.

24. Binder S, Knill T, Schuster J. Branched-chain amino acid metabolism in higher plants. Physiol Plant. 2007;129:68-78.

25. Joshi V, Joung JG, Fei Z, Jander G. Interdependence of threonine, methionine and isoleucine metabolism in plants: accumulation and transcriptional regulation under abiotic stress. Amino Acids. 2010;39:933-47.
26. Brunetti C, Guidi L, Sebastiani F, Tattini M. Isoprenoids and phenylpropanoids are key components of the antioxidant defense system of plants facing severe excess light stress. Environ Exp Bot. 2015;119:54-62.

27. Upchurch RG. Fatty acid unsaturation, mobilization, and regulation in the response of plants to stress. Biotechnol Lett. 2008;30:967-77.

28. Walters D, Raynor L, Mitchell A, Walker R, Walker K. Antifungal activities of four fatty acids against plant pathogenic fungi. Mycopathologia. 2004;157: 87-90.

29. del Baño MJ, Lorente J, Castillo J, Benavente-García O, Marín MP, Del Río JA et al. Flavonoid distribution during the development of leaves, flowers, stems, and roots of Rosmarinus officinalis. postulation of a biosynthetic pathway. J Agric Food Chem. 2004;52:4987-92.

30. Hadacek F. Secondary metabolites as plant traits: current assessment and future perspectives. Crit Rev Plant Sci. 2002;21:273-322.

31. Park J, Suh DH, Singh D, Lee S, Lee JS, Lee CH. Systematic metabolic profiling and bioactivity assays for bioconversion of Aceraceae family. PLoS One. 2018;13:e0198739.

32. Han DH, Lee MJ, Kim JH. Antioxidant and apoptosis-inducing activities of ellagic acid. Anticancer Res. 2006;26:3601-6.

33. Seckin B, Sekmen AH, Türkan I. An enhancing effect of exogenous mannitol on the antioxidant enzyme activities in roots of wheat under salt stress. J Plant Growth Regul. 2009:28:12.

34. lacopini P, Baldi M, Storchi P, Sebastiani L. Catechin, epicatechin, quercetin, rutin and resveratrol in red grape: Content, in vitro antioxidant activity and interactions. J Food Compos Anal. 2008;21:589-98.

35. Nakashima S, Matsuda H, Oda Y, Nakamura S, Xu F, Yoshikawa M. Melanogenesis inhibitors from the desert plant Anastatica hierochuntica in B16 melanoma cells. Bioorgan Med Chem. 2010;18:2337-45.

36. Villano D, Fernández-Pachón MS, Moyá ML, Troncoso AM. Radical scavenging ability of polyphenolic compounds towards DPPH free radical. Talanta. 2007:71:230-5.

\section{Publisher's Note}

Springer Nature remains neutral with regard to jurisdictional claims in published maps and institutional affiliations.
Ready to submit your research? Choose BMC and benefit from:

- fast, convenient online submission

- thorough peer review by experienced researchers in your field

- rapid publication on acceptance

- support for research data, including large and complex data types

- gold Open Access which fosters wider collaboration and increased citations

- maximum visibility for your research: over $100 \mathrm{M}$ website views per year

At $\mathrm{BMC}$, research is always in progress.

Learn more biomedcentral.com/submissions 